\title{
Amplitude Matching for Multizone Sound Field Control
}

This paper was downloaded from TechRxiv (https://www.techrxiv.org).

\section{LICENSE}

CC BY 4.0

SUBMISSION DATE / POSTED DATE

03-02-2022 / 07-02-2022

\section{CITATION}

Abe, Takumi; Koyama, Shoichi; Ueno, Natsuki; Saruwatari, Hiroshi (2022): Amplitude Matching for Multizone Sound Field Control. TechRxiv. Preprint. https://doi.org/10.36227/techrxiv.19115729.v1

DOI 


\title{
Amplitude Matching for Multizone Sound Field Control
}

\author{
Takumi Abe, Shoichi Koyama, Member, IEEE, Natsuki Ueno, Member, IEEE, \\ and Hiroshi Saruwatari, Member, IEEE
}

\begin{abstract}
A multizone sound field control method, called amplitude matching, is proposed. The objective of amplitude matching is to synthesize a desired amplitude (or magnitude) distribution over a target region with multiple loudspeakers, whereas the phase distribution is arbitrary. Most of the current multizone sound field control methods are intended to synthesize a specific sound field including phase or to control acoustic potential energy inside the target region. In amplitude matching, a specific desired amplitude distribution can be set, ignoring sound propagation directions. Although the optimization problem of amplitude matching does not have a closed-form solution, our proposed algorithm based on the alternating direction method of multipliers (ADMM) allows us to accurately and efficiently synthesize the desired amplitude distribution. We also introduce the differential-norm penalty for a time-domain filter design with a small filter length. The experimental results indicated that the proposed method outperforms current multizone sound field control methods in terms of accuracy of the synthesized amplitude distribution.
\end{abstract}

Index Terms-multizone sound field control, personal audio, pressure matching, amplitude matching

\section{INTRODUCTION}

$\mathbf{S}$ YNTHESIZING a desired sound field inside a target region using multiple loudspeakers (secondary sources) has various applications, such as virtual/augmented reality, spatial noise cancellation, and personal sound zone generation. Sound field synthesis/control methods can be classified into two categories. One includes methods based on boundary integral equations analytically derived from the Helmholtz equation, such as wave field synthesis and higher-order ambisonics [1][6]. The other includes methods based on numerical optimization to minimize error between synthesized and desired sound fields inside the target region, such as pressure matching (PM) and (weighted) mode matching [2], [7]-[10]. Since these numerical methods enable us to generate complex sound fields with a flexible array geometry of loudspeakers, they have a broad range of practical applications.

Multizone sound field control [11]-[15], which is one particular problem in sound field control, aims at generating different sound fields inside multiple target regions for personal audio applications [16], [17]. For example, planewave fields with different propagation angles are synthesized inside two regions. However, this problem is sometimes impractical because its physical feasibility becomes significantly low depending on the desired propagation angles of planewaves.

The authors are with the Graduate School of Information Science and Technology, the University of Tokyo, Tokyo 113-8656, Japan e-mail: (see https://www.sp.ipc.i.u-tokyo.ac.jp/members).

Manuscript received April XX, 20XX; revised August XX, 20XX.
On the other hand, in some applications, it is desired to generate sound fields of certain acoustic power levels rather than synthesizing specific sound fields such as planewave fields of particular propagation angles. For example, the acoustic power should be high in one region, but suppressed in another region, focusing on whether the sound is audible or not. In previous studies, this type of problem has been addressed as an acoustic contrast control (ACC) problem [18], [19], where the ratio of acoustic potential energy in one region to that in another region is maximized to generate acoustic bright and dark zones. However, the power distribution inside the target region cannot be controlled by ACC because only the total energy is taken into account. Furthermore, ACC cannot be applied to generating sound fields in more than two regions at different acoustic potential energies.

We consider the problem of synthesizing specific amplitude (or magnitude) distributions inside the target regions by using secondary sources, which is referred to as amplitude matching. For example, a flat amplitude distribution over a target region is set as a desired distribution but its phase distribution is left arbitrary. Such an optimization problem is neither linear nor convex; therefore, nonlinear optimization algorithms are necessary [20]. However, because of the indifferentiability of the cost function of amplitude matching, general gradient methods, such as gradient descent and (quasi-)Newton's methods, cannot be applied in a strict sense. Even if these algorithms work in practice, putting the theoretical validity aside, they basically require a high computational load for computing the inverse of a Hessian matrix (or its proxy) or searching step size parameter at each iteration.

In our previous study [21], we proposed amplitude matching based on the majorization-minimization (MM) algorithm [22], [23], where the optimization problem of amplitude matching is efficiently solved in the frequency domain with a guarantee of a monotonic nonincrease in the cost function. However, this algorithm tends to be stagnated at local minima or saddle points. Furthermore, solving the amplitude matching problem at each frequency can lead to discontinuities in phase between frequency bins. As a result, in a broadband case, the timedomain filter to obtain driving signals of the secondary sources can be unnecessarily long.

We propose amplitude matching based on the alternating direction method of multipliers (ADMM) [24] to achieve a more accurate synthesis of the amplitude distribution with an efficient procedure. We also introduce the differential-norm penalty to prevent discontinuities between frequency bins in the broadband case. The proposed amplitude matching method 
is evaluated by numerical experiments and compared with current multizone sound field control methods and amplitude matching based on MM algorithm. In addition, results of experiments using real data obtained in a practical environment are also presented.

The rest of this paper is organized as follows. In Section II, prior works on multizone sound field control methods are briefly summarized. The problem statement on amplitude matching is described with current algorithms for solving it in Section III. In Section IV, the proposed ADMM-based algorithm is developed. Differential-norm penalty for a timedomain filter design is introduced in Section V. Experimental results in the narrowband and broadband cases are presented in Section VI. Finally, Section VII concludes this paper.

\section{A. Notation}

Italic letters denote scalars, lower case boldface italic letters denote vectors, and upper case boldface italic letters denote matrices. The sets of real and complex numbers are denoted by $\mathbb{R}$ and $\mathbb{C}$, respectively. Subscripts of scalars, vectors, and matrices indicate their indexes. To illustrate, $x_{i, j}$ is the $(i, j)$ th entry of the matrix $\boldsymbol{X}$.

The imaginary unit and Napier's constant are denoted by $\mathrm{j}:=\sqrt{-1}$ and e, respectively. The complex conjugate, transpose, conjugate transpose, and inverse are denoted by superscripts $(\cdot)^{*},(\cdot)^{\mathrm{T}},(\cdot)^{\mathrm{H}}$, and $(\cdot)^{-1}$, respectively. The absolute value of a scalar $x$ is denoted by $|x|$. The argument of complex-valued scalar $x$ is denoted by $\arg (x)$. The signs $|\cdot|$ and $\arg (\cdot)$, and exponential e are also applied to vectors for each element. For example, $|\boldsymbol{x}| \odot \mathrm{e}^{\mathrm{j} \arg (\boldsymbol{y})}$ means the vector consisting of $\left|x_{i}\right| \mathrm{e}^{\mathrm{j} \arg \left(y_{i}\right)}$ with the Hadamard product $\odot$. The Euclid norm of a vector $\boldsymbol{x}$ is denoted by $\|\boldsymbol{x}\|$.

The angular frequency, sound velocity, and wavenumber are denoted by $\omega, c$, and $k=\omega / c$, respectively. The harmonic time dependence $\mathrm{e}^{-\mathrm{j} \omega t}$ with the time $t$ is assumed according to conventions.

\section{Prior Works on SOUnd Field Control}

Sound field control is aimed to synthesize a desired sound field inside a target region. Driving signals of $L$ secondary sources are controlled so that the synthesized sound field corresponds to the desired one inside the target region $\Omega$. The secondary sources are arbitrarily placed in the region outside $\Omega$, as shown in Fig. 1. The target region $\Omega$ can consist of multiple non-overlapped regions. The synthesized sound pressure $u^{\text {syn }}(\boldsymbol{r}, \omega)$ of angular frequency $\omega$ at position $\boldsymbol{r} \in \Omega$ is represented by a linear combination of transfer functions of the secondary sources as

$$
u^{\mathrm{syn}}(\boldsymbol{r}, \omega)=\sum_{l=1}^{L} d_{l}(\omega) g_{l}(\boldsymbol{r}, \omega),
$$

where $d_{l}(\omega)$ and $g_{l}(\boldsymbol{r}, \omega)$ are the driving signal and the transfer function (Green's function) at $r$ for the $l$ th secondary source $(l \in\{1, \ldots, L\})$, respectively. Hereafter, the angular frequency $\omega$ is omitted for notational simplicity.

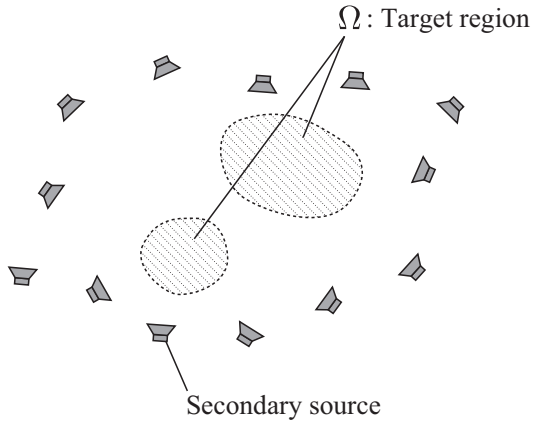

Fig. 1. Sound field control in multiple target regions.

The objective of general sound field control is to obtain the driving signals $\boldsymbol{d}=\left[d_{1}, \ldots, d_{L}\right]^{\top} \in \mathbb{C}^{L}$ so that the following cost function $F(\boldsymbol{d})$ is minimized:

$$
F(\boldsymbol{d}):=\int_{\Omega}\left|\sum_{l=1}^{L} d_{l} g_{l}(\boldsymbol{r})-u^{\mathrm{des}}(\boldsymbol{r})\right|^{2} \mathrm{~d} \boldsymbol{r},
$$

where $u^{\text {des }}(\boldsymbol{r})$ is the desired sound field. When $\Omega$ is separated into multiple regions, and different $u^{\mathrm{des}}(\boldsymbol{r})$ values are set inside each region, this minimization problem is specifically called multizone sound field control. Since minimizing (2) is difficult to solve owing to the regional integral, several approximation methods have been proposed.

\section{A. Pressure matching}

Pressure matching is a widely used sound field control method since its practical implementation is simple and flexible. The cost function $F(\boldsymbol{d})$ is approximated by discretizing the target region $\Omega$ into control points [7], [10], [11]. The number of control points and the position of the $m$ th control point are denoted by $M$ and $\boldsymbol{r}_{m} \in \Omega(m \in\{1, \ldots, M\})$, respectively. We define the vector $\boldsymbol{u}^{\text {des }} \in \mathbb{C}^{M}$ consisting of $u^{\mathrm{des}}\left(\boldsymbol{r}_{m}\right)$ and the matrix $\boldsymbol{G} \in \mathbb{C}^{M \times L}$ consisting of $g_{l}\left(\boldsymbol{r}_{m}\right)$. Thus, the optimization problem for the pressure matching is formulated as

$$
\underset{\boldsymbol{d}}{\operatorname{minimize}} F_{\mathrm{PM}}(\boldsymbol{d}):=\left\|\boldsymbol{G} \boldsymbol{d}-\boldsymbol{u}^{\mathrm{des}}\right\|^{2}+\gamma\|\boldsymbol{d}\|^{2},
$$

where $\gamma$ is a constant parameter. The first term is the square error between the synthesized and desired sound pressures at the control points, and the second term is the regularization term to prevent an excessive amplitude of $\boldsymbol{d}$. Since the control points should be densely placed inside $\Omega, M$ is generally larger than $L$. This optimization problem is solved in a closed form as the regularized least-squares solution:

$$
\hat{\boldsymbol{d}}=\left(\boldsymbol{G}^{\mathrm{H}} \boldsymbol{G}+\gamma \boldsymbol{I}\right)^{-1} \boldsymbol{G}^{\mathrm{H}} \boldsymbol{u}^{\mathrm{des}} .
$$

The pressure-matching method can be directly applied to the multizone sound field control.

Instead of discretizing the target region $\Omega$, the sound field is represented by spherical wave function expansion in modematching methods [2]. The driving signal $\boldsymbol{d}$ is obtained so that the expansion coefficients of $u^{\mathrm{syn}}(\boldsymbol{r})$ correspond to those of $u^{\text {des }}(\boldsymbol{r})$. The weighting factors for each expansion coefficient are introduced in the weighted mode-matching [9] 
for accurate approximation of the original cost function (2) without empirical truncation of the expansion order. The mode-matching methods are also extended to multizone sound field control [12], [13].

\section{B. Acoustic contrast control}

Generating regions of high- and low-acoustic power distributions is one particular example of the multizone sound field control. In ACC [18], [19], the target region $\Omega$ is separated into two regions $\Omega_{\mathrm{B}}$ and $\Omega_{\mathrm{D}}\left(\Omega_{\mathrm{B}} \cup \Omega_{\mathrm{D}}=\Omega\right.$ and $\left.\Omega_{\mathrm{B}} \cap \Omega_{\mathrm{D}}=\emptyset\right)$. The regions $\Omega_{\mathrm{B}}$ and $\Omega_{\mathrm{D}}$ are the so-called (acoustic) bright and dark zones, respectively. The goal is to synthesize high acoustic potential energy inside $\Omega_{\mathrm{B}}$ and low acoustic potential energy inside $\Omega_{\mathrm{D}}$.

The optimization problem of ACC is defined as the maximization of the ratio of acoustic potential energy inside $\Omega_{\mathrm{B}}$ to that inside $\Omega_{\mathrm{D}}$ as

$$
\underset{\boldsymbol{d}}{\operatorname{maximize}} Q(\boldsymbol{d}):=\frac{\left\|\boldsymbol{G}_{\mathrm{B}} \boldsymbol{d}\right\|^{2}}{\left\|\boldsymbol{G}_{\mathrm{D}} \boldsymbol{d}\right\|^{2}}=\frac{\boldsymbol{d}^{\mathrm{H}} \boldsymbol{G}_{\mathrm{B}}^{\mathrm{H}} \boldsymbol{G}_{\mathrm{B}} \boldsymbol{d}}{\boldsymbol{d}^{\mathrm{H}} \boldsymbol{G}_{\mathrm{D}}^{\mathrm{H}} \boldsymbol{G}_{\mathrm{D}} \boldsymbol{d}},
$$

where $\boldsymbol{G}_{\mathrm{B}}$ and $\boldsymbol{G}_{\mathrm{D}}$ are the transfer function matrices at the control points inside $\Omega_{\mathrm{B}}$ and $\Omega_{\mathrm{D}}$, respectively. The optimal $\boldsymbol{d}$ can be obtained as the eigenvector that corresponds to the largest eigenvalue of the matrix

$$
\left(\boldsymbol{G}_{\mathrm{D}}^{\mathrm{H}} \boldsymbol{G}_{\mathrm{D}}+\mu \boldsymbol{I}\right)^{-1} \boldsymbol{G}_{\mathrm{B}}^{\mathrm{H}} \boldsymbol{G}_{\mathrm{B}},
$$

where $\mu$ is the regularization parameter. This solution has indefiniteness for the scaling and phase rotation of $\boldsymbol{d}$. Since only the total acoustic energy inside the target region is considered in (5), it is not possible to synthesize specific power distributions. Therefore, a large variation of acoustic power inside the bright zone can be generated. Furthermore, ACC cannot be applied to controlling more than two regions with different acoustic power levels.

\section{Amplitude MATCHING}

Pressure matching is applicable to synthesizing various desired sound fields inside the target region. However, in some applications, it is necessary to synthesize a sound field of the desired amplitude inside the target region, whereas the phase of the desired sound field is arbitrary. For example, the sound field of high- and low-acoustic-power distributions should be synthesized similarly to that in ACC. We formulate the optimization problem to achieve sound field control only with the amplitude constraint, i.e., amplitude matching, as

$$
\underset{\boldsymbol{d}}{\operatorname{minimize}} J(\boldsymbol{d}):=\left\||\boldsymbol{G} \boldsymbol{d}|-\left|\boldsymbol{u}^{\mathrm{des}}\right|\right\|^{2}+\lambda\|\boldsymbol{d}\|^{2},
$$

where $\lambda$ is the constant parameter. The first term is the square error between the synthesized and desired amplitude distributions at the control points, and the second term is the regularization term of $\boldsymbol{d}$. In contrast to the cost function of ACC (5), (7) is designed to measure the error of spatial amplitude distributions. Thus, various amplitude distributions can be set as the desired sound field in the amplitude matching.

Since the objective function of the amplitude matching $J(\boldsymbol{d})$ is neither linear nor convex, nonlinear optimization algorithms are necessary to solve (7). Gradient methods, such as gradient descent and (quasi-)Newton's method, are widely used nonlinear optimization algorithms. Since $J(\boldsymbol{d})$ is not differentiable at an arbitrary point, the gradient methods are not applicable to solving (7) in a strict sense, but they can work in practice. The gradient of $J(\boldsymbol{d})$ can be obtained as

$$
\nabla J(\boldsymbol{d})=\boldsymbol{G}^{\mathrm{H}}\left(|\boldsymbol{G} \boldsymbol{d}|-\left|\boldsymbol{u}^{\mathrm{des}}\right|\right) \odot \mathrm{e}^{\mathrm{j} \arg (\boldsymbol{G d})}+\lambda \boldsymbol{d},
$$

where $\boldsymbol{G} \boldsymbol{d}$ is assumed not to contain zero. By using (8), we can construct the gradient methods for solving (7), such as gradient descent and quasi-Newton's methods. In addition to the lack of theoretical validity, the computational complexity of these algorithms can be high, as discussed later.

\section{A. MM algorithm}

An amplitude matching method based on the MM algorithm has been proposed by Koyama et al. [21]. In the MM algorithm, a surrogate function for a nonconvex objective function, which can be simply minimized, is constructed. The monotonic nonincrease in the objective function can be guaranteed by alternately updating the variable of the surrogate function and the variable to be optimized. The resulting update rules can be computationally efficient by appropriately constructing the surrogate function.

In [21], the surrogate function of $J(\boldsymbol{d})$ is obtained as

$$
J^{+}\left(\boldsymbol{d} \mid \boldsymbol{v}^{(i)}\right):=\left\|\boldsymbol{G} \boldsymbol{d}-\boldsymbol{v}^{(i)}\right\|^{2}+\lambda\|\boldsymbol{d}\|^{2},
$$

where $\boldsymbol{v}^{(i)}$ is the auxiliary variable defined with the iteration index $i$ as

$$
\boldsymbol{v}^{(i)}:=\left|\boldsymbol{u}^{\mathrm{des}}\right| \odot \mathrm{e}^{\mathrm{j} \arg \left(\boldsymbol{G} \boldsymbol{d}^{(i)}\right)} .
$$

Here, $\boldsymbol{d}^{(i)}$ is $\boldsymbol{d}$ at the $i$ th iteration. This surrogate function satisfies

$$
J(\boldsymbol{d}) \leq J^{+}\left(\boldsymbol{d} \mid \boldsymbol{v}^{(i)}\right)
$$

where the equality holds for $\boldsymbol{d}=\boldsymbol{d}^{(i)}$. See Appendix A for the proof. Thus, the driving signal $\boldsymbol{d}$ is obtained by alternately updating $\boldsymbol{v}^{(i)}$ and $\boldsymbol{d}^{(i)}$ as

$$
\begin{aligned}
\boldsymbol{v}^{(i)} & =\left|\boldsymbol{u}^{\mathrm{des}}\right| \odot \mathrm{e}^{\mathrm{j} \arg \left(\boldsymbol{G} \boldsymbol{d}^{(i)}\right)}, \\
\boldsymbol{d}^{(i+1)} & =\underset{\boldsymbol{d}}{\arg \min } J^{+}\left(\boldsymbol{d} \mid \boldsymbol{v}^{(i)}\right) \\
& =\left(\boldsymbol{G}^{\mathrm{H}} \boldsymbol{G}+\lambda \boldsymbol{I}\right)^{-1} \boldsymbol{G}^{\mathrm{H}} \boldsymbol{v}^{(i)} .
\end{aligned}
$$

The monotonic nonincrease in the objective function can be confirmed as

$$
J\left(\boldsymbol{d}^{(i+1)}\right) \leq J^{+}\left(\boldsymbol{d}^{(i+1)} \mid \boldsymbol{v}^{(i)}\right) \leq J^{+}\left(\boldsymbol{d}^{(i)} \mid \boldsymbol{v}^{(i)}\right)=J\left(\boldsymbol{d}^{(i)}\right) .
$$

The iterative update rules are computed until a stopping condition is satisfied, for example, by setting a threshold for the variation of $J\left(\boldsymbol{d}^{(i)}\right)$ or $\boldsymbol{d}^{(i)}$. The MM algorithm for the amplitude matching is summarized in Algorithm 1. Note that $\left(\boldsymbol{G}^{\mathrm{H}} \boldsymbol{G}+\lambda \boldsymbol{I}\right)^{-1} \boldsymbol{G}^{\mathrm{H}}$ in the fourth line can be calculated before the iteration. Thus, the computational cost for each iteration is $O(L M)$. This algorithm corresponds to the Griffin-Lim algorithm [25] and Gerchberg-Saxton algorithm [26] for the phase retrieval problem, whose cost function is similar to that in (7). 


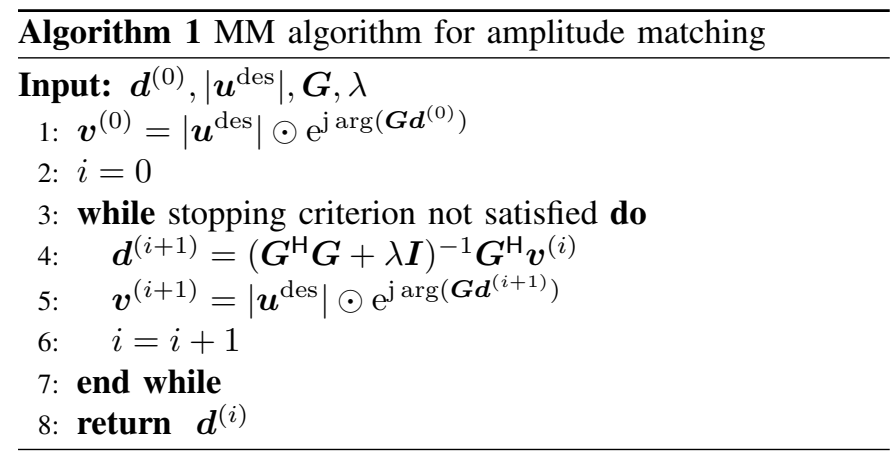

\section{Alternating Direction Method for Multipliers FOR AMPLitude Matching}

The MM algorithm presented in Section III-A is computationally efficient and simple for implementation. However, in some cases, the cost function does not sufficiently decrease, as later shown in the experiments. We propose the amplitude matching algorithm based on ADMM. For convex optimization problems, the convergence to the optimal solution can be guaranteed by ADMM [24]. It has been experimentally validated that ADMM effectively works for some nonconvex optimization problems [24], [27], [28]. In particular, Liang et al. [27] have shown that ADMM performs well in phase retrieval problems.

Since ADMM cannot be directly applied to solve (7), we reformulate the optimization problem by using auxiliary variables of amplitude and phase defined as $\boldsymbol{G d}=\boldsymbol{a} \odot \mathrm{e}^{\mathrm{j} \boldsymbol{\theta}}$ as

$$
\begin{aligned}
& \underset{\boldsymbol{d}, \boldsymbol{a} \geq \mathbf{0}, \boldsymbol{\theta}}{\operatorname{minimize}}\left\|\boldsymbol{a}-\left|\boldsymbol{u}^{\mathrm{des}}\right|\right\|^{2}+\lambda\|\boldsymbol{d}\|^{2} \\
& \text { subject to } \boldsymbol{G} \boldsymbol{d}=\boldsymbol{a} \odot \mathrm{e}^{\mathrm{j} \boldsymbol{\theta}},
\end{aligned}
$$

where $\boldsymbol{a} \geq 0$ means that each element of $\boldsymbol{a}$ is equal to or larger than 0 . Then, the augmented Lagrangian function for (15) can be defined as

$$
\begin{aligned}
& \quad L_{\rho}(\boldsymbol{d}, \boldsymbol{a}, \boldsymbol{\theta}, \boldsymbol{w}):=\left\|\boldsymbol{a}-\left|\boldsymbol{u}^{\mathrm{des}}\right|\right\|^{2}+\lambda\|\boldsymbol{d}\|^{2} \\
& \quad+\boldsymbol{w}^{\mathrm{H}}\left(\boldsymbol{G} \boldsymbol{d}-\boldsymbol{a} \odot \mathrm{e}^{\mathrm{j} \boldsymbol{\theta}}\right)+\frac{\rho}{2}\left\|\boldsymbol{G} \boldsymbol{d}-\boldsymbol{a} \odot \mathrm{e}^{\mathrm{j} \boldsymbol{\theta}}\right\|^{2},
\end{aligned}
$$

where $\boldsymbol{w} \in \mathbb{C}^{M}$ is the Lagrange multiplier and $\rho>0$ is the penalty parameter. In ADMM, each variable is alternately updated, starting with initial values as

$$
\begin{aligned}
& \left\{\boldsymbol{a}^{(i+1)}, \boldsymbol{\theta}^{(i+1)}\right\}=\underset{\boldsymbol{a} \geq \mathbf{0}, \boldsymbol{\theta}}{\arg \min } L_{\rho}\left(\boldsymbol{d}^{(i)}, \boldsymbol{a}, \boldsymbol{\theta}, \boldsymbol{w}^{(i)}\right), \\
& \boldsymbol{d}^{(i+1)}=\underset{\boldsymbol{d}}{\arg \min } L_{\rho}\left(\boldsymbol{d}, \boldsymbol{a}^{(i+1)}, \boldsymbol{\theta}^{(i+1)}, \boldsymbol{w}^{(i)}\right), \\
& \boldsymbol{w}^{(i+1)}=\boldsymbol{w}^{(i)}+\rho\left(\boldsymbol{G} \boldsymbol{d}^{(i+1)}-\boldsymbol{a}^{(i+1)} \odot \mathrm{e}^{\mathrm{j} \boldsymbol{\theta}^{(i+1)}}\right) .
\end{aligned}
$$

The augmented Lagrangian function for each update is minimized for one or two variables while fixing the other variables.
To obtain the update rules of $\boldsymbol{a}$ and $\boldsymbol{\theta}$, we modify the subproblem (17) as

$$
\begin{aligned}
& \left\{\boldsymbol{a}^{(i+1)}, \boldsymbol{\theta}^{(i+1)}\right\} \\
& =\underset{\boldsymbol{a} \geq \mathbf{0}, \boldsymbol{\theta}}{\arg \min } L_{\rho}\left(\boldsymbol{d}^{(i)}, \boldsymbol{a}, \boldsymbol{\theta}, \boldsymbol{w}^{(i)}\right) \\
& =\underset{\boldsymbol{a} \geq \mathbf{0}, \boldsymbol{\theta}}{\arg \min }\left\|\boldsymbol{a}-\left|\boldsymbol{u}^{\mathrm{des}}\right|\right\|^{2}+\lambda\left\|\boldsymbol{d}^{(i)}\right\|^{2}+\frac{\rho}{2}\left\|\boldsymbol{h}^{(i)}-\boldsymbol{a} \odot \mathrm{e}^{\mathrm{j} \boldsymbol{\theta}}\right\|^{2},
\end{aligned}
$$

where $\boldsymbol{h}^{(i)}=\boldsymbol{G} \boldsymbol{d}^{(i)}+\boldsymbol{w}^{(i)} / \rho$. The phase $\boldsymbol{\theta}$ is included only in the third term, and $\boldsymbol{\theta}^{(i+1)}$ is obviously determined regardless of $\boldsymbol{a}$. Thus, the third term of (20) is minimized with respect to $\boldsymbol{\theta}$ by setting it to the phase of $\boldsymbol{h}^{(i)}$ as

$$
\boldsymbol{\theta}^{(i+1)}=\arg \left(\boldsymbol{h}^{(i)}\right)
$$

Then, $\boldsymbol{a}$ is updated by minimizing the quadratic of $\boldsymbol{a}$ as

$$
\begin{aligned}
& \boldsymbol{a}^{(i+1)}=\underset{\boldsymbol{a} \geq \mathbf{0}}{\arg \min }\left\|\boldsymbol{a}-\left|\boldsymbol{u}^{\mathrm{des}}\right|\right\|^{2}+\frac{\rho}{2}\left\|\left|\boldsymbol{h}^{(i)}\right|-\boldsymbol{a}\right\|^{2} \\
& =\frac{\rho\left|\boldsymbol{h}^{(i)}\right|+2\left|\boldsymbol{u}^{\mathrm{des}}\right|}{\rho+2} \text {. }
\end{aligned}
$$

The update rule of $\boldsymbol{d}$ is obtained by solving

$$
\begin{aligned}
& \boldsymbol{d}^{(i+1)} \\
& =\underset{\boldsymbol{d}}{\arg \min } L_{\rho}\left(\boldsymbol{d}, \boldsymbol{a}^{(i+1)}, \boldsymbol{\theta}^{(i+1)}, \boldsymbol{w}^{(i)}\right) \\
& =\underset{\boldsymbol{d}}{\arg \min } \lambda\|\boldsymbol{d}\|^{2}+\frac{\rho}{2}\left\|\boldsymbol{G d}+\frac{\boldsymbol{w}^{(i)}}{\rho}-\boldsymbol{a}^{(i+1)} \odot \mathrm{e}^{\mathrm{j}^{(i+1)}}\right\|^{2},
\end{aligned}
$$

where the same modification as that in (20) is used. This quadratic of $\boldsymbol{d}$ can also be simply minimized as

$$
\begin{aligned}
& \boldsymbol{d}^{(i+1)}= \\
& \left(\frac{2 \lambda}{\rho} \boldsymbol{I}+\boldsymbol{G}^{\mathrm{H}} \boldsymbol{G}\right)^{-1} \boldsymbol{G}^{\mathrm{H}}\left(\boldsymbol{a}^{(i+1)} \odot \mathrm{e}^{\mathrm{j} \boldsymbol{\theta}^{(i+1)}}-\frac{\boldsymbol{w}^{(i)}}{\rho}\right) .
\end{aligned}
$$

Note that the inverse matrix in (24) can be computed before the iteration.

We summarize the proposed ADMM-based amplitude matching algorithm in Algorithm 2. The computational cost of the proposed algorithm for each iteration is $O(L M)$, which is identical to that of the MM-based algorithm. Among the gradient methods, the computational cost of the gradient descent method is relatively small, which requires $O(L M)$ for computing the gradient (8) and the cost for the line search of the step-size parameter. However, its convergence speed is generally low. The quasi-Newton's methods, such as the Broyden-Fletcher-Goldfarb-Shanno (BFGS) method, are practically useful because of their fast convergence and low computational cost by approximating the inverse of the Hessian matrix. The computational cost for each iteration is $O\left(L^{2}+L M\right)$ with the line search of the step-size parameter. Therefore, the proposed method is computationally efficient as the MM-based algorithm. Further investigation is given in the experiments. 


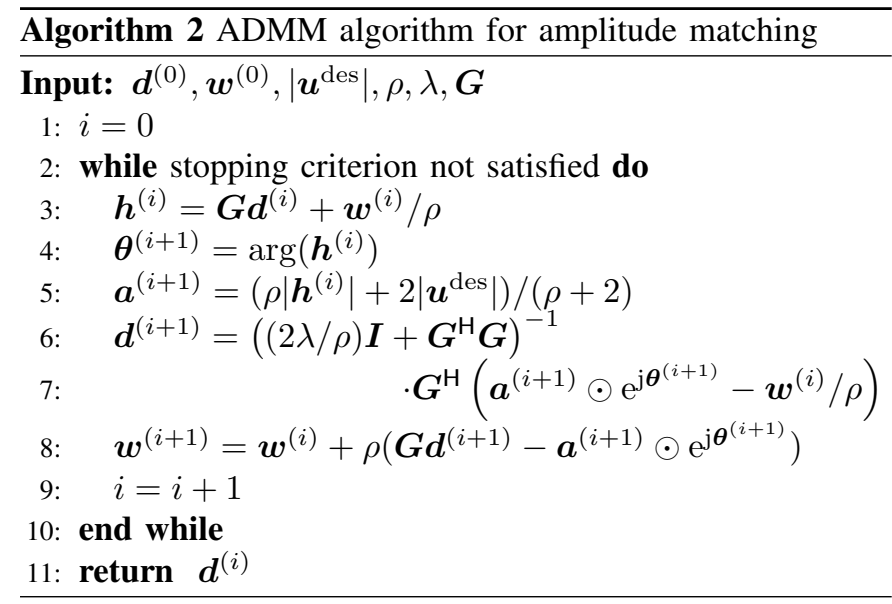

\section{Differential-Norm Penalty for Time-Domain FILTER Design}

In the previous section, the optimal driving signals for amplitude matching are obtained in the frequency domain. In practical implementations, the frequency-domain driving signals of target frequency bins are transformed into the time domain by inverse Fourier transform to construct a singleinput-multiple-output (SIMO) finite impulse response (FIR) filter, where the input is the source signal for playback and the output is the driving signals of the secondary sources. The procedure to obtain broadband driving signals is equivalent to that to solve the following optimization problem:

$$
\underset{\left\{\boldsymbol{d}_{k}\right\}_{k=1}^{K}}{\operatorname{minimize}} \sum_{k=1}^{K}\left\|\left|\boldsymbol{G}_{k} \boldsymbol{d}_{k}\right|-\left|\boldsymbol{u}^{\mathrm{des}}\right|\right\|^{2}+\lambda \sum_{k=1}^{K}\left\|\boldsymbol{d}_{k}\right\|^{2},
$$

where the index of the frequency bin $k(\in\{1, \ldots, K\})$ is introduced in the subscript for each frequency-dependent variable. Here, $\left|\boldsymbol{u}^{\mathrm{des}}\right|$ is assumed to be independent of frequency to obtain a flat amplitude response filter for each control point. Since the loss term and the regularization term of $\ell_{2}$-norm for each frequency are simply added, (25) can be solved at every frequency as in Section IV.

However, since the phase of the driving signals is independently determined for each frequency, the resulting solution can have discontinuities between frequencies. These discontinuities can lead to an unnecessarily large FIR filter length. To achieve amplitude matching with a small FIR filter length, we propose the use of a penalty term for the discontinuities of $\boldsymbol{d}_{k}$, which is called the differential-norm penalty. We define the differential norm as

$$
D\left(\boldsymbol{d}_{k}\right):=\sum_{k=2}^{K}\left\|\boldsymbol{d}_{k}-\boldsymbol{d}_{k-1}\right\|^{2}=\|\overline{\boldsymbol{F}} \overline{\boldsymbol{d}}\|^{2},
$$

where $\overline{\boldsymbol{d}}=\left[\boldsymbol{d}_{1}^{\top}, \ldots, \boldsymbol{d}_{K}^{\top}\right]^{\top} \in \mathbb{C}^{L K}$ and $\overline{\boldsymbol{F}} \in \mathbb{C}^{L(K-1) \times L K}$ is defined as

$$
\overline{\boldsymbol{F}}:=\left[\begin{array}{cccccccc}
-1 & 0 & \cdots & 0 & 1 & & & \\
& -1 & 0 & & 0 & 1 & & \boldsymbol{O} \\
& & \ddots & \ddots & & \ddots & \ddots & \\
& \boldsymbol{O} & & -1 & 0 & \cdots & 0 & 1
\end{array}\right] .
$$

Thus, the optimization problem of the amplitude matching with the differential-norm penalty is described as

$$
\underset{\left\{\boldsymbol{d}_{k}\right\}_{k=1}^{K}}{\operatorname{minimize}} \sum_{k=1}^{K}\left\|\left|\boldsymbol{G}_{k} \boldsymbol{d}_{k}\right|-\left|\boldsymbol{u}^{\mathrm{des}}\right|\right\|^{2}+\lambda\|\overline{\boldsymbol{F}} \overline{\boldsymbol{d}}\|^{2} .
$$

The optimization problem (28) can be solved by ADMM as in the single-frequency case. First, (28) is reformulated as

$$
\begin{aligned}
& \underset{\overline{\boldsymbol{d}}, \overline{\boldsymbol{a}} \geq \mathbf{0}, \overline{\boldsymbol{\theta}}}{\operatorname{minimize}}\left\|\overline{\boldsymbol{a}}-\left|\overline{\boldsymbol{u}}^{\mathrm{des}}\right|\right\|^{2}+\lambda\|\overline{\boldsymbol{F}} \overline{\boldsymbol{d}}\|^{2} \\
& \text { subject to } \overline{\boldsymbol{G}} \overline{\boldsymbol{d}}=\overline{\boldsymbol{a}} \odot \mathrm{e}^{\mathrm{j} \overline{\boldsymbol{\theta}}},
\end{aligned}
$$

where $\overline{\boldsymbol{a}}=\left[\boldsymbol{a}_{1}^{\top}, \ldots, \boldsymbol{a}_{K}^{\top}\right]^{\top} \in \mathbb{R}_{>0}^{M K}, \overline{\boldsymbol{\theta}}=\left[\boldsymbol{\theta}_{1}^{\top}, \ldots, \boldsymbol{\theta}_{K}^{\top}\right]^{\top} \in$ $\mathbb{R}^{M K},\left|\overline{\boldsymbol{u}}^{\mathrm{des}}\right|=\left[\left|\boldsymbol{u}^{\mathrm{des}}\right|^{\top}, \ldots,\left|\boldsymbol{u}^{\mathrm{des}}\right|^{\top}\right]^{\top} \in \mathbb{R}_{>0}^{M K}$, and $\overline{\boldsymbol{G}} \in$ $\mathbb{C}^{M K \times L K}$ is the block diagonal matrix of $\left\{\boldsymbol{G}_{k}\right\}_{k=1}^{K}$ as

$$
\bar{G}:=\left[\begin{array}{ccc}
G_{1} & & O \\
& \ddots & \\
O & & G_{K}
\end{array}\right] .
$$

The augmented Lagrangian function is defined with the Lagrange multiplier $\overline{\boldsymbol{w}}=\left[\boldsymbol{w}_{1}^{\top}, \ldots, \boldsymbol{w}_{K}^{\top}\right]^{\top} \in \mathbb{C}^{M K}$ as

$$
\begin{aligned}
& \bar{L}_{\rho}(\overline{\boldsymbol{d}}, \overline{\boldsymbol{a}}, \overline{\boldsymbol{\theta}}, \overline{\boldsymbol{w}}):=\left\|\overline{\boldsymbol{a}}-\left|\overline{\boldsymbol{u}}^{\mathrm{des}}\right|\right\|^{2}+\lambda\|\overline{\boldsymbol{F}} \overline{\boldsymbol{d}}\|^{2} \\
& \quad+\overline{\boldsymbol{w}}^{\mathrm{H}}\left(\overline{\boldsymbol{G}} \overline{\boldsymbol{d}}-\overline{\boldsymbol{a}} \odot \mathrm{e}^{\mathrm{j} \overline{\boldsymbol{\theta}}}\right)+\frac{\rho}{2}\left\|\overline{\boldsymbol{G}} \overline{\boldsymbol{d}}-\overline{\boldsymbol{a}} \odot \mathrm{e}^{\mathrm{j} \overline{\boldsymbol{\theta}}}\right\|^{2} .
\end{aligned}
$$

The update rule of each variable is obtained as

$$
\begin{aligned}
& \overline{\boldsymbol{\theta}}^{(i+1)}=\arg \left(\overline{\boldsymbol{h}}^{(i)}\right), \\
& \overline{\boldsymbol{a}}^{(i+1)}=\frac{\rho\left|\overline{\boldsymbol{h}}^{(i)}\right|+2\left|\overline{\boldsymbol{u}}^{\mathrm{des}}\right|}{\rho+2}, \\
& \overline{\boldsymbol{d}}^{(i+1)}=\boldsymbol{M}^{-1} \overline{\boldsymbol{G}}^{\mathrm{H}}\left(\overline{\boldsymbol{a}}^{(i+1)} \odot \mathrm{e}^{\mathrm{j} \overline{\boldsymbol{\theta}}^{(i+1)}}-\frac{\overline{\boldsymbol{w}}^{(i)}}{\rho}\right), \\
& \overline{\boldsymbol{w}}^{(i+1)}=\overline{\boldsymbol{w}}^{(i)}+\rho\left(\overline{\boldsymbol{G}} \overline{\boldsymbol{d}}^{(i+1)}-\overline{\boldsymbol{a}}^{(i+1)} \odot \mathrm{e}^{\mathrm{j} \overline{\boldsymbol{\theta}}^{(i+1)}}\right),
\end{aligned}
$$

where $\overline{\boldsymbol{h}}^{(i)}=\overline{\boldsymbol{G}} \overline{\boldsymbol{d}}^{(i)}+\overline{\boldsymbol{w}}^{(i)} / \rho\left(\in \mathbb{C}^{M K}\right)$, and $\boldsymbol{M} \in \mathbb{C}^{L K \times L K}$ is defined as

$$
\boldsymbol{M}=\left[\begin{array}{ccc}
\boldsymbol{M}_{11} & \ldots & \boldsymbol{M}_{K 1} \\
\vdots & \ddots & \vdots \\
\boldsymbol{M}_{1 K} & \ldots & \boldsymbol{M}_{K K}
\end{array}\right]
$$

with

$$
\boldsymbol{M}_{i j}=\left\{\begin{array}{ll}
\boldsymbol{G}_{i}^{\mathrm{H}} \boldsymbol{G}_{i}+\frac{2 \lambda}{\rho} \boldsymbol{I} & i=j=1, K \\
\boldsymbol{G}_{i}^{\mathrm{H}} \boldsymbol{G}_{i}+\frac{4 \lambda}{\rho} \boldsymbol{I} & i=j \neq 1, K \\
-\frac{2 \lambda}{\rho} \boldsymbol{I} & i=j+1, j-1 \\
\boldsymbol{O} & \text { otherwise }
\end{array} .\right.
$$

The update rules of $\overline{\boldsymbol{\theta}}, \overline{\boldsymbol{a}}$, and $\overline{\boldsymbol{w}}$ are efficiently computed. On the other hand, the update of $\bar{d}$ in (34) requires large computational complexity owing to $M^{-1}$. Since the computation of $\boldsymbol{M}^{-1} \bar{G}^{\mathrm{H}}$ remains unchanged for each iteration, which can be performed before the iteration, which requires $O\left((M+L) L^{2} K^{3}\right)$, then the computational cost of the update of $\overline{\boldsymbol{d}}$ becomes $O\left(L M K^{2}\right)$. However, these computational complexities can be reduced by using the property of the block 
tridiagonal matrix of $\boldsymbol{M}$. We apply the block birecurrence method [29] in updating $\overline{\boldsymbol{d}}$, which reduces the computational cost to $O\left(L^{3} K\right)$ before the iteration and $O((M+L) L K)$ for each iteration. Furthermore, the memory requirements can also be reduced from $O\left((M+L) L K^{2}\right)$ to $O((M+L) L K)$ before the iteration and from $O\left(M L K^{2}\right)$ to $O((M+L) L K)$ at each iteration. Since $K$ is generally much larger than $L$ and $M$, these reductions of temporal and spatial complexities are significant. See Appendix B for the detailed procedure of the block birecurrence method.

\section{EXPERIMENTS}

We conducted experiments to evaluate our proposed method. First, experimental results in the narrowband case are shown to compare the performance of the amplitude matching algorithms. Next, the broaband case is investigated to validate the effectiveness of the differential norm. Finally, we demonstrate experimental results using real data.

\section{A. Narrowband case}

We conducted numerical experiments using 2D free-field simulations in the frequency domain, focusing on the comparison of the amplitude matching (AM) algorithms described in Section III with the conventional pressure matching (PM) and ACC. As shown in Fig. 2, a circular loudspeaker array was placed, whose radius was $1.5 \mathrm{~m}$ and the center was at the origin. The array consisted of 48 loudspeakers that were equiangularly aligned. Each loudspeaker was assumed to be the $2 \mathrm{D}$ point source, whose transfer function is defined as

$$
g_{l}(\boldsymbol{r})=\frac{\mathrm{j}}{4} H_{0}^{(1)}\left(k\left\|\boldsymbol{r}-\boldsymbol{r}_{l}\right\|\right),
$$

where $H_{0}^{(1)}(\cdot)$ is the 0th-order Hankel function of the first kind and $\boldsymbol{r}_{l}$ is the position of the $l$ th loudspeaker. Two circular target regions were set inside the loudspeaker array. The radii of the two regions were both $0.3 \mathrm{~m}$, and their centers were at $(0.0,0.5) \mathrm{m}$ and $(0.0,-0.5) \mathrm{m}$. The upper and lower target regions are denoted as $\Omega_{\mathrm{U}}$ and $\Omega_{\mathrm{L}}$, respectively. The target regions were discretized into 111 control points at intervals of $0.05 \mathrm{~m}$. For the desired amplitude distributions, we set 1.0 in $\Omega_{\mathrm{U}}$ and 0.0 in $\Omega_{\mathrm{L}}$; therefore, $\Omega_{\mathrm{U}}$ and $\Omega_{\mathrm{L}}$ are supposed to be acoustic bright and dark zones, respectively. Hence, ACC was applied to synthesizing bright and dark zones in each region. The amplitude of the driving signal for ACC was determined so that the average amplitude inside $\Omega_{\mathrm{U}}$ becomes 1.0. PM was applied by setting $\boldsymbol{u}^{\text {des }}$ in (3) as 1.0 in $\Omega_{\mathrm{U}}$ and 0.0 in $\Omega_{\mathrm{L}}$.

To solve the optimization problem of AM, we compared the proposed ADMM-based algorithm (AM-ADMM), MMbased algorithm (AM-MM), and gradient method using BFGS (AM-BFGS). PM and ACC were also evaluated. Since the experiments were conducted in the frequency domain, we used the frequency-domain ADMM algorithm (Algorithm 2) in this section. The initial value of $\boldsymbol{d}$ for the three AM algorithms was the solution of PM. The regularization parameter $\lambda$ in (7) for the AM algorithms and $\gamma$ in (5) for ACC were set as $\sigma_{\max }(\boldsymbol{G}) \times 10^{-3}$, where $\sigma_{\max }(\boldsymbol{G})$ means the largest singular value of $\boldsymbol{G}$. The parameter $\rho$ in Algorithm 2 was set to 1.0.

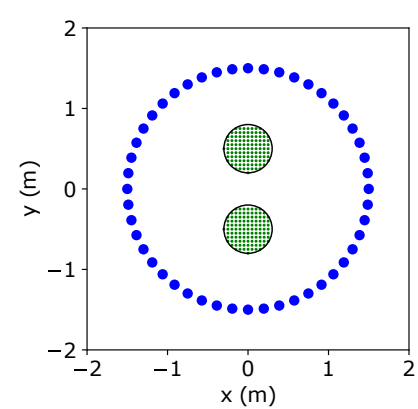

Fig. 2. Experimental setting for narrowband and broadband cases. Blue and green dots indicate loudspeakers and control points, respectively.

As an evaluation measure, we define the mean square error (MSE) between synthesized and desired amplitude distributions as

$$
\operatorname{MSE}(\omega)=10 \log _{10}\left(\frac{1}{M}\left\|\left|\boldsymbol{u}^{\mathrm{syn}}(\omega)\right|-\left|\boldsymbol{u}^{\mathrm{des}}(\omega)\right|\right\|^{2}\right),
$$

where $\left|\boldsymbol{u}^{\mathrm{syn}}(\omega)\right|$ is the amplitude distribution of the synthesized sound field. The experiments were conducted using a 3.00 GHz 8-Core Intel Core i7-9700F processor and implemented with Python.

Fig. 3 shows the MSE of each method with respect to the frequency from 100 to $1000 \mathrm{~Hz}$ at intervals of $50 \mathrm{~Hz}$. Since the optimization problem of AM was solved for each frequency, the MSEs of the AM algorithms largely varied at each frequency. However, they were significantly lower than those of PM and ACC. Among the three AM algorithms, the MSEs of AM-MM were relatively higher than those of AM-ADMM and AM-BFGS, especially at the frequencies below $1100 \mathrm{~Hz}$. This means that AM-MM did not reach the optimal solution owing to its initial value dependence at these frequencies. The performance of AM-ADMM was comparable to that of AM-BFGS, but high MSEs appeared at several frequencies. Nevertheless, their general performance was better than that of the other methods.

The synthesized pressure distribution at $1100 \mathrm{~Hz}$ of each method is shown in Fig. 4. Table I shows the MSE of each method. The MSE of PM was particularly high because synthesizing a uniform pressure distribution of zero phase is impractical. In ACC, a low acoustic potential energy was achieved in $\Omega_{\mathrm{L}}$, but the amplitude distribution in $\Omega_{\mathrm{U}}$ was not uniform. Thus, even though the MSE of ACC was very low in $\Omega_{\mathrm{L}}$, that of ACC was high in $\Omega_{\mathrm{U}}$. The plane-wavelike pressure distributions were synthesized by AM-BFGS and AM-ADMM, although their angles were slightly different. In AM-MM, the synthesized pressure distribution was still close to that in PM, i.e., the initial value. The total MSE was the lowest in AM-ADMM.

Fig. 5 shows the cost function value of the AM algorithms in terms of computation time. The decreasing speed of the cost function was high in AM-MM and AM-ADMM, but it stagnated at around 7.5 in AM-MM. In AM-ADMM and AMBFGS, the cost function decreased to 0.31. The computation time until the cost function reached 0.31 was $55.4 \mathrm{~ms}$ in 


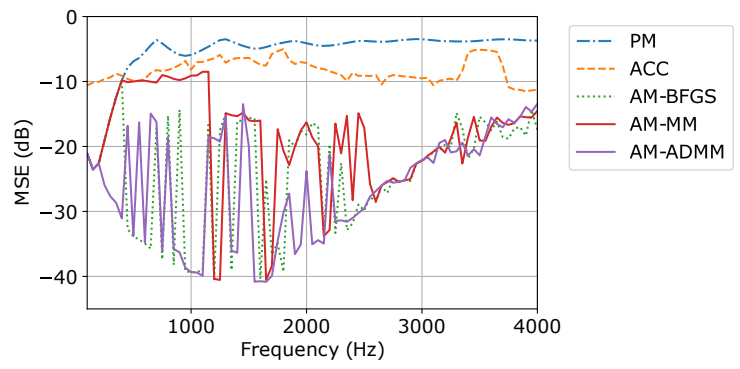

Fig. 3. MSE with respect to frequency for narrowband case.

TABLE I

MSE IN dB IN NARROWBAND CASE AT $1100 \mathrm{~Hz}$.

\begin{tabular}{lccc}
\hline & MSE & MSE in $\Omega_{\mathrm{U}}$ & MSE in $\Omega_{\mathrm{L}}$ \\
\hline \hline PM & -5.1 & -2.1 & -26.5 \\
ACC & -7.0 & -4.0 & $-\mathbf{6 8 . 1}$ \\
AM-BFGS & -39.1 & -36.5 & -46.5 \\
AM-MM & -8.5 & -5.6 & -21.4 \\
AM-ADMM & $-\mathbf{3 9 . 9}$ & $-\mathbf{3 7 . 1}$ & -49.5 \\
\hline
\end{tabular}

AM-BFGS and $12.2 \mathrm{~ms}$ in AM-ADMM. A similar tendency can be seen at other frequencies. Therefore, the proposed ADMM algorithm is effective both in control accuracy and computational time.

Next, we demonstrate the case that the desired amplitude distributions are more complex. We set three circular target regions of $0.3 \mathrm{~m}$ radius with their centers at $(-0.2 \sqrt{3}, 0.2) \mathrm{m}$, $(0.2 \sqrt{3}, 0.2) \mathrm{m}$, and $(0.0,-0.4) \mathrm{m}$. We denote these upper left, upper right, and lower center target regions as $\Omega_{\mathrm{UL}}, \Omega_{\mathrm{UR}}$, and $\Omega_{\mathrm{LC}}$, respectively. The desired amplitude distributions were 1.0 in $\Omega_{\mathrm{UL}}, 0.5$ in $\Omega_{\mathrm{UR}}$, and 0.0 in $\Omega_{\mathrm{LC}}$. In this case, ACC is not applicable since the ratio of acoustic potential energies cannot be defined for the three regions. MSE at $800 \mathrm{~Hz}$ is shown in Table II. The synthesized pressure distributions of three AM methods are shown in Fig. 6. These results indicate that our proposed algorithm performs well even when the desired amplitude distributions are complex.

\section{B. Broadband case}

Next, we show experimental results in the broadband case, focusing on the effectiveness validation of the differentialnorm penalty for AM. Again, numerical simulation is conducted under the 2D free-field assumption. The placements of loudspeakers, target regions, and control points, and the settings of the desired amplitude were the same as those in Section VI-A (see Fig. 2).

We compare AM with the differential-norm penalty (28) and $\ell_{2}$-norm regularization (25), which are denoted as AM w/ Diff.-norm and $\mathrm{AM} w / \ell_{2}$-norm, respectively. The parameter $\lambda$ in (28) was set to 10.0 , and the other parameters were the same as those in AM-ADMM in the narrowband case. After generating the frequency-domain driving signals using the ADMM-based algorithm, time-domain FIR filters were obtained by their inverse fast Fourier transform (FFT). The sampling frequency was $16 \mathrm{kHz}$, and the number of frequency bins, i.e., FFT length, was 32768. As an evaluation measure,

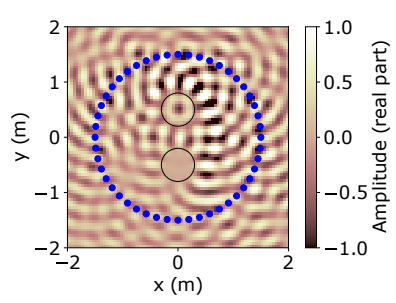

(a)

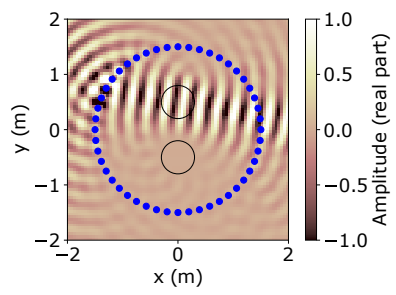

(c)

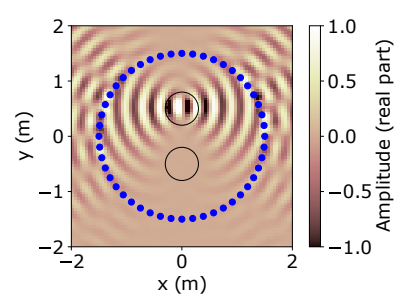

(b)

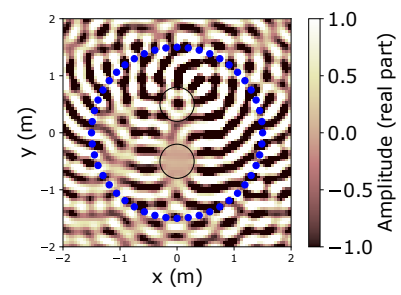

(d)

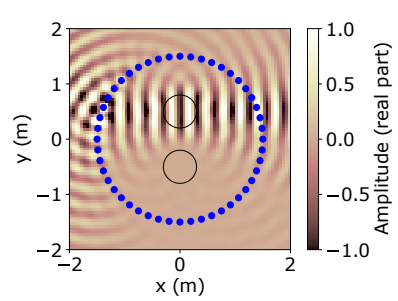

(e)

Fig. 4. Synthesized pressure distribution in narrowband case at $1100 \mathrm{~Hz}$. (a) PM; (b) ACC; (c) AM-BFGS; (d) AM-MM; (e) AM-ADMM.

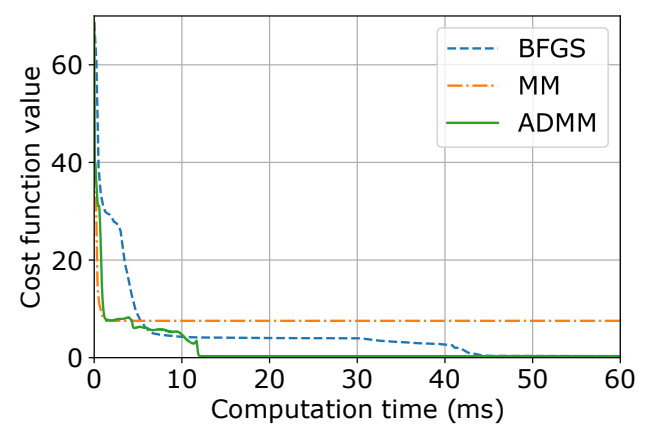

Fig. 5. Cost function value with respect to computation time. Three AM algorithms were compared.

MSE was calculated in the time domain using time-domain amplitudes of synthesized and desired sound fields at each evaluation point, $\tilde{\boldsymbol{a}}^{\text {syn }}$ and $\tilde{\boldsymbol{a}}^{\text {des }}$, as

$$
\mathrm{MSE}=10 \log _{10}\left(\frac{1}{M}\left\|\tilde{\boldsymbol{a}}^{\mathrm{syn}}-\tilde{\boldsymbol{a}}^{\mathrm{des}}\right\|^{2}\right) .
$$

Here, the time-domain amplitude is computed as

$$
\tilde{a}_{m}^{\mathrm{syn}}=\sqrt{\sum_{n=1}^{N} u_{m}^{\mathrm{syn}}(n)^{2}},
$$

where $u_{m}^{\mathrm{syn}}(n)$ is the synthesized pressure of time index $n$ $(\in\{1, \ldots, N\})$ at the $m$ th evaluation point. 
TABLE II

MSE IN dB FOR NARROWBAND CASE AT $800 \mathrm{HZ}$ WHEN THREE TARGET REGIONS WERE SET.

\begin{tabular}{lcccc}
\hline & MSE & MSE in $\Omega_{\mathrm{UL}}$ & MSE in $\Omega_{\mathrm{UR}}$ & MSE in $\Omega_{\mathrm{LC}}$ \\
\hline \hline PM & -5.3 & -1.5 & -7.6 & -25.6 \\
AM-BFGS & -16.6 & -12.9 & -20.0 & $-\mathbf{2 3 . 1}$ \\
AM-MM & -10.3 & -6.9 & -12.6 & -17.3 \\
AM-ADMM & $\mathbf{- 2 1 . 5}$ & $\mathbf{- 2 1 . 0}$ & $\mathbf{- 2 0 . 6}$ & -22.7 \\
\hline
\end{tabular}

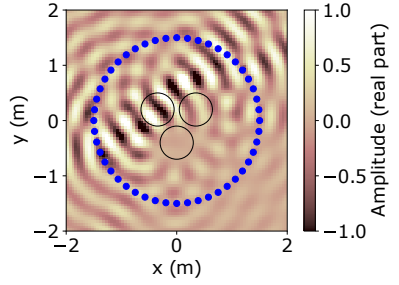

(a)

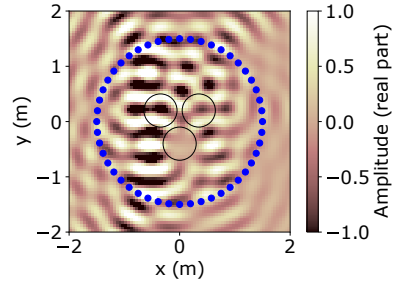

(b)

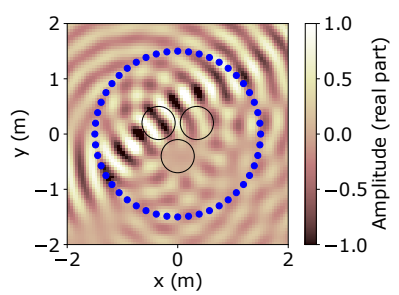

(c)

Fig. 6. Synthesized pressure distribution of three AM methods in narrowband case at $800 \mathrm{~Hz}$ when three target regions were set. (a) AM-BFGS; (b) AMMM; (c) AM-ADMM.

Fig. 7 shows the FIR filters of AM w/ Diff.-norm and $\ell_{2}$-norm before truncating the filter length. Apparently, the decrease in the filter amplitude from the peak of the AM w/ Diff.-norm is much sharper than that of $\ell_{2}$-norm. When the optimal filter length is defined so that the decrease from the peak amplitude is $30 \mathrm{~dB}$, that of $\mathrm{AM}$ w/ $\ell_{2}$-norm was 13682 . On the other hand, the optimal filter length of AM w/ Diff.norm was 314.

Table III shows the MSE of each method when the filter length is truncated to 684. This filter length is defined so that the decrease in filter amplitude from the peak is $40 \mathrm{~dB}$ in AM w/ Diff.-norm. MSE in the frequency domain is also plotted in Fig. 8. The time-domain MSE of AM w/ Diff.-norm was particularly lower than that of $\ell_{2}$-norm inside $\Omega_{\mathrm{U}}$. The frequency-domain MSE of AM w/ $\ell_{2}$-norm largely fluctuated for each frequency bin at high frequencies, whereas that of Diff.-norm was relatively smooth.

\section{Experiments using real data}

To investigate the performance of the proposed method in a practical environment, we conducted experiments using the impulse response dataset presented in [30]. Configurations of loudspeakers and control points are shown in Fig. 9. We set two 2D square target regions of acoustic bright and dark zones, $\Omega_{\mathrm{B}}$ and $\Omega_{\mathrm{D}}$, with dimensions of $0.4 \mathrm{~m} \times 0.3 \mathrm{~m}$ on the $x y$ plane at $z=0$, whose centers were at $(0.0 \mathrm{~m}, 0.4 \mathrm{~m}, 0.0 \mathrm{~m})$

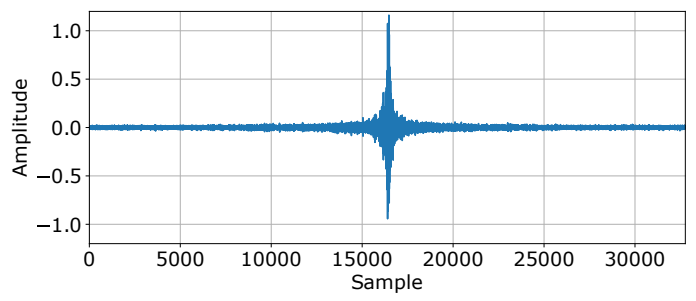

(a)

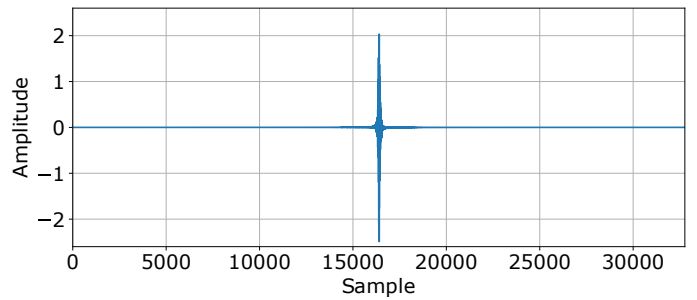

(b)

Fig. 7. Time-domain filters generated by (a) AM w/ $\ell_{2}$-norm and (b) AM w/ Diff.-norm.

TABLE III

MSE IN dB IN BROADBAND CASE.

\begin{tabular}{lccc}
\hline & MSE & MSE in $\Omega_{\mathrm{U}}$ & MSE in $\Omega_{\mathrm{L}}$ \\
\hline \hline AM w/ Diff.-norm & $\mathbf{- 1 9 . 5}$ & $\mathbf{- 2 1 . 7}$ & -18.0 \\
AM w/ $\ell_{2}$-norm & -18.3 & -18.1 & $\mathbf{- 1 8 . 4}$ \\
\hline
\end{tabular}

and $(0.0 \mathrm{~m},-0.4 \mathrm{~m}, 0.0 \mathrm{~m})$, respectively. The loudspeakers were regularly arranged along the borders of two squares with dimensions of $2.0 \mathrm{~m} \times 2.0 \mathrm{~m}$ at $z= \pm 0.1 \mathrm{~m}$. Twenty control points were obtained by discretizing each target region every $0.1 \mathrm{~m}$. The desired amplitudes were 1.0 in $\Omega_{\mathrm{B}}$ and 0.0 in $\Omega_{\mathrm{D}}$ and the generated signal was a band-limited pulse signal from $100 \mathrm{~Hz}$ to $1000 \mathrm{~Hz}$. MSE in the time domain was evaluated at the positions of $0.05 \mathrm{~m}$ intervals inside the target regions. The reverberation time $T_{60}$ was $0.19 \mathrm{~s}$.

First, the filters generated by $\mathrm{AM}$ w/ $\ell_{2}$-norm and Diff.norm are compared. The parameters for ADMM-based algorithms were the same as those in Section VI-B. Fig. 10 shows the filters before truncation obtained by $\mathrm{AM}$ w/ $\ell_{2}$-norm and Diff.-norm. Since the impulse responses of the loudspeakers include reverberation, the difference in the necessary filter length for these two methods was much larger than that in Section VI-B.

Table IV shows the MSE in the time domain of each method. The proposed AM w/ Diff.-norm achieved the lowest MSE among the four methods. In particular, the MSE in $\Omega_{\mathrm{B}}$ of the proposed method was $10 \mathrm{~dB}$ lower than those of the other methods. The power distributions of the synthesized sound field, i.e., $\left(\tilde{\boldsymbol{a}}^{\mathrm{syn}}\right)^{2}$, are shown in Fig. 11 . In $\Omega_{\mathrm{D}}$, the power distribution of the proposed method was low even at the positions other than the control points, compared with those of the other methods.

\section{CONCLUSION}

We proposed the amplitude matching method for multizone sound field control. The amplitude matching aims to synthesize 


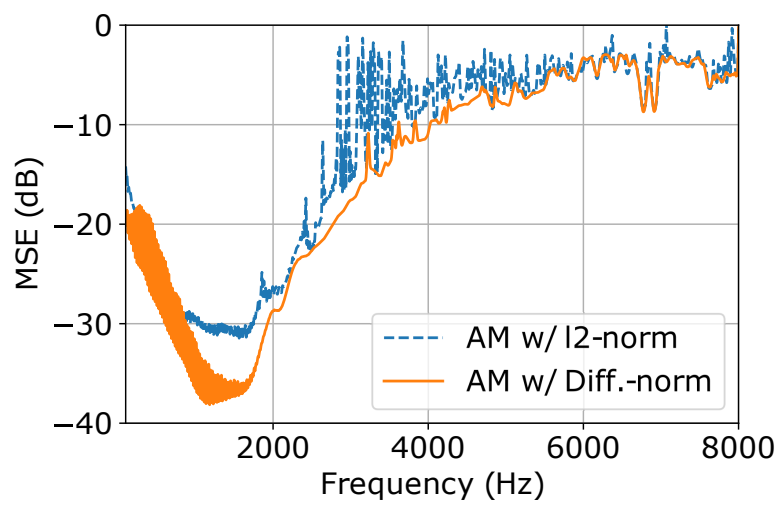

Fig. 8. MSE with respect to frequency in broadband case.

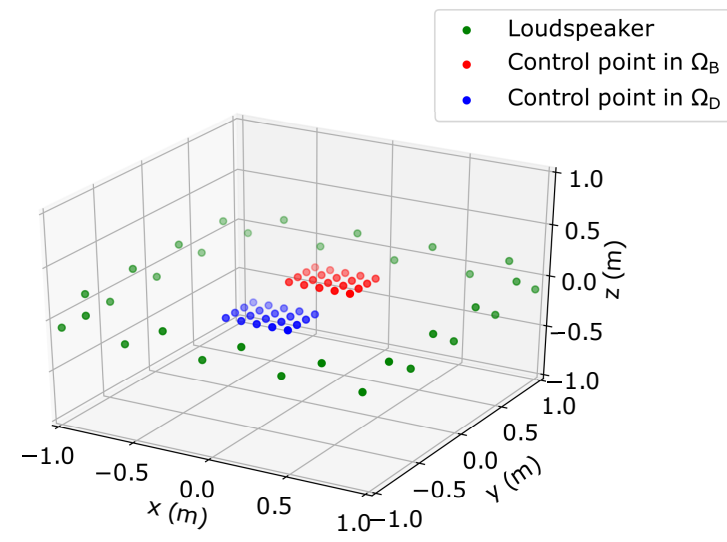

Fig. 9. Placements of loudspeakers and control points in the experiments using real data. Green, red, and blue dots indicate loudspeakers, control points in $\Omega_{\mathrm{B}}$, and those in $\Omega_{\mathrm{D}}$, respectively.

a desired amplitude distribution over a target region, whereas the phase distribution is arbitrary, using multiple secondary sources. Since the cost function of amplitude matching is neither linear, convex, nor differentiable, it is important to develop efficient algorithms for solving the amplitude matching problem. An algorithm based on ADMM is formulated both in the frequency and time domains. To avoid an unnecessarily large time-domain filter length in the broadband case, the differential-norm penalty for adjacent frequency bins is also introduced. In the experiments, it was shown that the proposed method enables us to accurately and efficiently synthesize the desired amplitude distributions, compared with current multizone sound field control methods.

\section{APPENDIX A}

\section{DERIVATION OF MM ALGORITHM FOR AMPLITUDE} MATCHING

By using

$$
(\boldsymbol{G} \boldsymbol{d})^{\mathrm{H}} \boldsymbol{v}^{(i)}=|\boldsymbol{G} \boldsymbol{d}|^{\top}\left|\boldsymbol{u}^{\mathrm{des}}\right| \odot \mathrm{e}^{\mathrm{j} \arg \left(\boldsymbol{G} \boldsymbol{d}^{(i)}-\arg (\boldsymbol{G} \boldsymbol{d})\right)},
$$

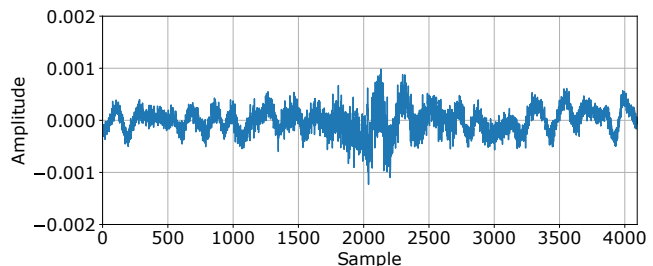

(a)

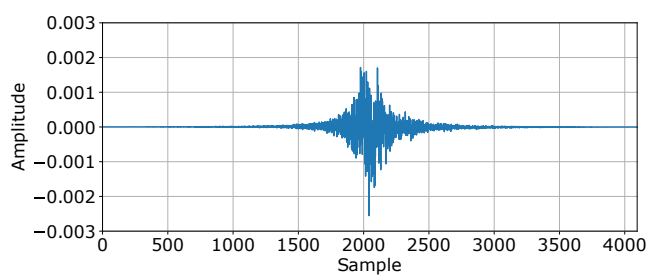

(b)

Fig. 10. Time-domain filters generated by (a) $\mathrm{AM} w / \ell_{2}$-norm and (b) $\mathrm{AM}$ w/ Diff.-norm when using real data.

TABLE IV

MSE IN dB FOR EXPERIMENTS USING REAL DATA.

\begin{tabular}{lccc}
\hline & MSE & MSE in $\Omega_{\mathrm{B}}$ & MSE in $\Omega_{\mathrm{D}}$ \\
\hline \hline AM w/ Diff. norm & $\mathbf{- 2 2 . 7}$ & $\mathbf{- 3 2 . 4}$ & $\mathbf{- 2 0 . 0}$ \\
$\mathrm{AM}$ w/ $\ell_{2}$ norm & -18.5 & -23.9 & -16.1 \\
$\mathrm{PM}$ & -13.0 & -20.1 & -10.4 \\
$\mathrm{ACC}$ & -16.1 & -14.9 & -17.7 \\
\hline
\end{tabular}

we obtain the following inequality:

$$
\begin{aligned}
(\boldsymbol{G d})^{\mathrm{H}} \boldsymbol{v}^{(i)} & +\boldsymbol{v}^{(i) \mathrm{H}} \boldsymbol{G d} \\
& =2|\boldsymbol{G d}|^{\top}\left|\boldsymbol{u}^{\mathrm{des}}\right| \odot \cos \left(\arg \left(\boldsymbol{G} \boldsymbol{d}^{(i)}\right)-\arg (\boldsymbol{G d})\right) \\
& \leq 2|\boldsymbol{G d}|^{\top}\left|\boldsymbol{u}^{\mathrm{des}}\right|,
\end{aligned}
$$

where the equality holds for $\boldsymbol{d}=\boldsymbol{d}^{(i)}$. Therefore,

$$
\begin{aligned}
\left\||\boldsymbol{G} \boldsymbol{d}|-\left|\boldsymbol{u}^{\mathrm{des}}\right|\right\|^{2} & =\|\boldsymbol{G} \boldsymbol{d}\|^{2}-2|\boldsymbol{G} \boldsymbol{d}|^{\top}\left|\boldsymbol{u}^{\mathrm{des}}\right|+\left\|\boldsymbol{u}^{\mathrm{des}}\right\|^{2} \\
& \leq\|\boldsymbol{G} \boldsymbol{d}\|^{2}-\boldsymbol{d}^{\mathrm{H}} \boldsymbol{G}^{\mathrm{H}} \boldsymbol{v}^{(i)}-\boldsymbol{v}^{(i) \mathrm{H}} \boldsymbol{G} \boldsymbol{d}+\left\|\boldsymbol{u}^{\mathrm{des}}\right\|^{2} \\
& =\left\|\boldsymbol{G} \boldsymbol{d}-\boldsymbol{v}^{(i)}\right\|^{2}
\end{aligned}
$$

Thus, we obtain the surrogate function of $J(\boldsymbol{d})$ as

$$
J^{+}\left(\boldsymbol{d} \mid \boldsymbol{v}^{(i)}\right)=\left\|\boldsymbol{G} \boldsymbol{d}-\boldsymbol{v}^{(i)}\right\|^{2}+\lambda\|\boldsymbol{d}\|^{2} .
$$

\section{APPENDIX B}

\section{BLOCK BIRECURRENCE METHOD FOR AMPLITUDE}

MATCHING WITH DIFFERENTIAL-NORM PENALTY

We apply the block birecurrence method [29] to solve (34) in ADMM algorithm for amplitude matching with the differential-norm penalty. First, (34) is rewritten as the block tridiagonal simultaneous linear equations as

$$
\boldsymbol{M}\left[\begin{array}{c}
\boldsymbol{d}_{1}^{(i+1)} \\
\boldsymbol{d}_{2}^{(i+1)} \\
\vdots \\
\vdots \\
\boldsymbol{d}_{K}^{(i+1)}
\end{array}\right]=\left[\begin{array}{c}
\boldsymbol{p}_{1}^{(i+1)} \\
\boldsymbol{p}_{2}^{(i+1)} \\
\vdots \\
\vdots \\
\boldsymbol{p}_{K}^{(i+1)}
\end{array}\right],
$$




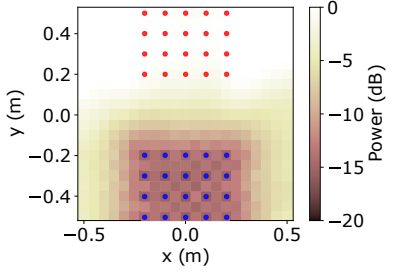

(a)

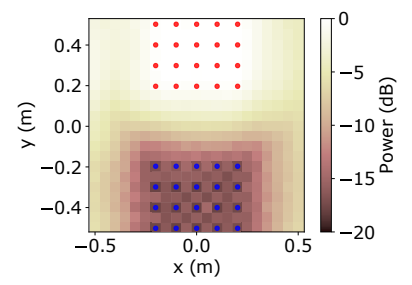

(c)

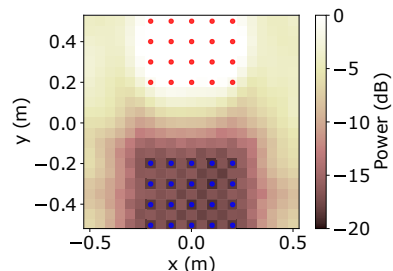

(b)

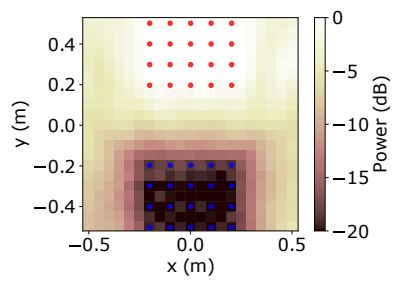

(d)
Fig. 11. Power distributions of synthesized sound fields when using real data Red and blue dots indicate control points in $\Omega_{\mathrm{B}}$ and $\Omega_{\mathrm{D}}$, respectively. (a) PM; (b) ACC; (c) AM w/ $\ell_{2}$-norm; (d) AM w/ Diff.-norm.

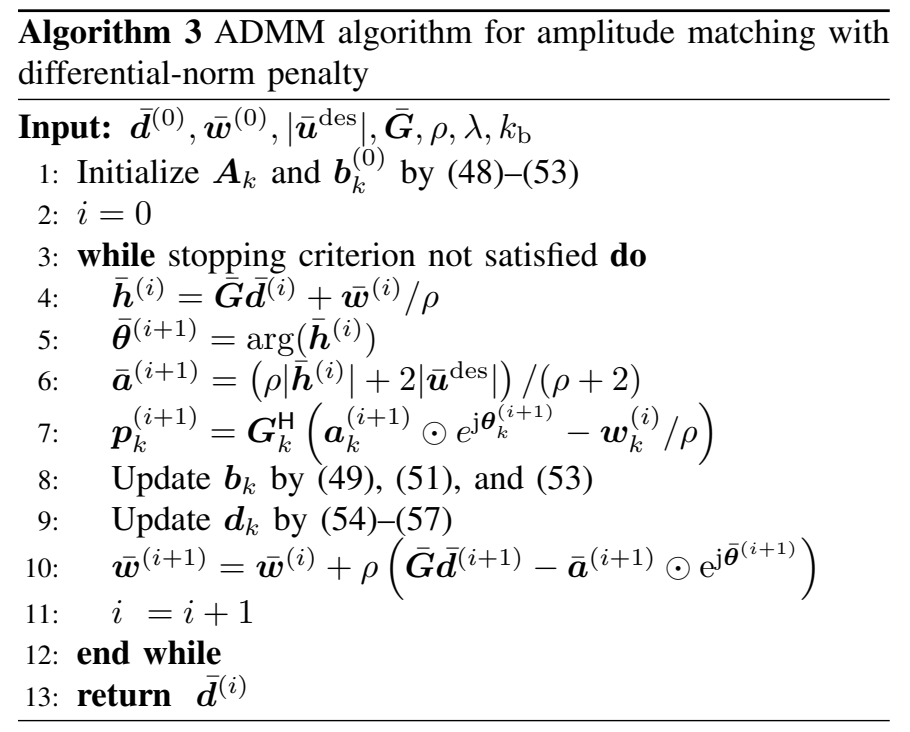

where $\boldsymbol{p}_{k}^{(i+1)}$ is defined as

$$
\boldsymbol{p}_{k}^{(i+1)}:=\boldsymbol{G}_{k}^{\mathrm{H}}\left(\boldsymbol{a}_{k}^{(i+1)} \odot \mathrm{e}^{\mathrm{j} \boldsymbol{\theta}_{k}^{(i+1)}}-\frac{\boldsymbol{w}_{k}^{(i)}}{\rho}\right)
$$

We define $\left\{\boldsymbol{A}_{k}\right\}_{k=1}^{K}$ and $\left\{\boldsymbol{b}_{k}\right\}_{k=1}^{K}$, which are separately computed for $k=1, k \in\left[2, k_{\mathrm{b}}\right], k \in\left[k_{\mathrm{b}}+1, K-1\right]$, and $k=K$ with the integer $k_{\mathrm{b}}$ (balancer). Their update rules are written as

$$
\begin{aligned}
\boldsymbol{A}_{k} & =\frac{2 \lambda}{\rho}\left(\boldsymbol{G}_{k}^{\mathrm{H}} \boldsymbol{G}_{k}+\frac{2 \lambda}{\rho} \boldsymbol{I}\right)^{-1}, \\
\boldsymbol{b}_{k}^{(i+1)} & =\left(\boldsymbol{G}_{k}^{\mathrm{H}} \boldsymbol{G}_{k}+\frac{2 \lambda}{\rho} \boldsymbol{I}\right)^{-1} \boldsymbol{p}_{k}^{(i+1)},
\end{aligned}
$$

for $k=1$ and $K$,

$$
\begin{aligned}
& \boldsymbol{A}_{k}=\frac{2 \lambda}{\rho}\left(\boldsymbol{G}_{k}^{\mathrm{H}} \boldsymbol{G}_{k}+\frac{4 \lambda}{\rho} \boldsymbol{I}-\frac{2 \lambda}{\rho} \boldsymbol{A}_{k-1}\right)^{-1}, \\
& \boldsymbol{b}_{k}^{(i+1)}= \\
& \left(\boldsymbol{G}_{k}^{\mathrm{H}} \boldsymbol{G}_{k}+\frac{4 \lambda}{\rho} \boldsymbol{I}-\frac{2 \lambda}{\rho} \boldsymbol{A}_{k-1}\right)^{-1}\left(\boldsymbol{p}_{k}^{(i+1)}+\frac{2 \lambda}{\rho} \boldsymbol{b}_{k-1}^{(i+1)}\right),
\end{aligned}
$$

for $k=2, \ldots, k_{\mathrm{b}}$, and

$$
\begin{aligned}
& \boldsymbol{A}_{k}=\frac{2 \lambda}{\rho}\left(\boldsymbol{G}_{k}^{\mathrm{H}} \boldsymbol{G}_{k}+\frac{4 \lambda}{\rho} \boldsymbol{I}-\frac{2 \lambda}{\rho} \boldsymbol{A}_{k+1}\right)^{-1}, \\
& \boldsymbol{b}_{k}^{(i+1)}= \\
& \left(\boldsymbol{G}_{k}^{\mathrm{H}} \boldsymbol{G}_{k}+\frac{4 \lambda}{\rho} \boldsymbol{I}-\frac{2 \lambda}{\rho} \boldsymbol{A}_{k+1}\right)^{-1}\left(\boldsymbol{p}_{k}^{(i+1)}+\frac{2 \lambda}{\rho} \boldsymbol{b}_{k+1}^{(i+1)}\right),
\end{aligned}
$$

for $k=K-1, K-2, \ldots, k_{\mathrm{b}}+1$. We set $k_{\mathrm{b}}$ to $K / 2$ in the experiment.

Then, the recursive update rule of $\left\{\boldsymbol{d}_{k}\right\}_{k=1}^{K}$ is obtained as

$$
\boldsymbol{d}_{k_{\mathrm{b}}}^{(i+1)}=\left(\boldsymbol{I}-\boldsymbol{A}_{k_{\mathrm{b}}} \boldsymbol{A}_{k_{\mathrm{b}}+1}\right)^{-1}\left(\boldsymbol{b}_{k_{\mathrm{b}}}^{(i+1)}+\boldsymbol{A}_{k_{\mathrm{b}}} \boldsymbol{b}_{k_{\mathrm{b}}+1}^{(i+1)}\right),
$$

for $k=k_{\mathrm{b}}$,

$$
\boldsymbol{d}_{k_{\mathrm{b}}+1}^{(i+1)}=\left(\boldsymbol{I}-\boldsymbol{A}_{k_{\mathrm{b}}+1} \boldsymbol{A}_{k_{\mathrm{b}}}\right)^{-1}\left(\boldsymbol{b}_{k_{\mathrm{b}}+1}^{(i+1)}+\boldsymbol{A}_{k_{\mathrm{b}}+1} \boldsymbol{b}_{k_{\mathrm{b}}}^{(i+1)}\right),
$$

for $k=k_{\mathrm{b}}+1$,

$$
\boldsymbol{d}_{k}^{(i+1)}=\boldsymbol{A}_{k} \boldsymbol{d}_{k+1}+\boldsymbol{b}_{k}^{(i+1)},
$$

for $k=k_{\mathrm{b}}-1, k_{\mathrm{b}}-2, \ldots, 1$, and

$$
\boldsymbol{d}_{k}^{(i+1)}=\boldsymbol{A}_{k} \boldsymbol{d}_{k-1}+\boldsymbol{b}_{k}^{(i+1)},
$$

for $k=k_{\mathrm{b}}+2, k_{\mathrm{b}}+3, \ldots, K$.

Since inverse matrices in the computation of $\left\{\boldsymbol{b}_{k}^{(i+1)}\right\}_{k=1}^{K}$, $\boldsymbol{d}_{k_{\mathrm{b}}}^{(i+1)}, \boldsymbol{d}_{k_{\mathrm{b}}+1}^{(i+1)}$, and $\left\{\boldsymbol{A}_{k}\right\}_{k=1}^{K}$ remain unchanged at each iteration, they can be computed before the iteration. The proposed ADMM algorithm for amplitude matching with the differential-norm penalty is summarized in Algorithm 3.

\section{ACKNOWLEDGMENT}

This work was supported by JST PRESTO Grant Number JPMJPR18J4.

\section{REFERENCES}

[1] S. Spors, R. Rabenstein, and J. Ahrens, "The theory of wave field synthesis revisited," in Proc. 124th AES Conv., Amsterdam, Netherlands, Oct. 2008.

[2] M. A. Poletti, "Three-dimensional surround sound systems based on spherical harmonics," J. Audio Eng. Soc., vol. 53, no. 11, pp. 1004 $1025,2005$.

[3] J. Ahrens and S. Spors, "An analytical approach to sound field reproduction using circular and spherical loudspeaker distributions," Acta Acust. united Ac., vol. 94, pp. 988-999, 2008.

[4] Y. J. Wu and T. D. Abhayapala, "Theory and design of soundfield reproduction using continuous loudspeaker concept," IEEE Trans. Audio, Speech, Lang. Process., vol. 17, no. 1, pp. 107-116, 2009.

[5] S. Koyama, K. Furuya, Y. Hiwasaki, and Y. Haneda, "Analytical approach to wave field reconstruction filtering in spatio-temporal frequency domain," IEEE Trans. Audio, Speech, Lang. Process., vol. 21, no. 4, pp. 685-696, 2013. 
[6] S. Koyama, K. Furuya, K. Wakayama, S. Shimauchi, and H. Saruwatari, "Analytical approach to transforming filter design for sound field recording and reproduction using circular arrays with a spherical baffle," $J$. Acoust. Soc. Amer., vol. 139, no. 3, pp. 1024-1036, 2016.

[7] O. Kirkeby, P. A. Nelson, F. O. Bustamante, and H. Hamada, "Local sound field reproduction using digital signal processing," J. Acoust. Soc. Amer., vol. 100, no. 3, pp. 1584-1593, 1996.

[8] T. Betlehem and T. D. Abhayapala, "Theory and design of sound field reproduction in reverberant environment," J. Acoust. Soc. Amer, vol. 117, no. 4, pp. 2100-2111, 2005.

[9] N. Ueno, S. Koyama, and H. Saruwatari, "Three-dimensional sound field reproduction based on weighted mode-matching method," IEEE/ACM Trans. Audio, Speech, Lang. Process., vol. 27, no. 12, pp. 1852-1867, 2019.

[10] S. Koyama, G. Chardon, and L. Daudet, "Optimizing source and sensor placement for sound field control: An overview," IEEE/ACM Trans. Audio, Speech, Lang. Process., vol. 28, pp. 686-714, 2020.

[11] M. Poletti, "An investigation of 2-D multizone surround sound systems," in Proc. 125th AES Conv., San Francisco, Oct. 2008.

[12] W. Zhang, T. Abhayapala, T. Betlehem, and F. Fazi, "Analysis and control of multi-zone sound field reproduction using modal-domain approach," J. Acoust. Soc. Amer, vol. 140, pp. 2134-2144, Sep. 2016.

[13] Y. J. Wu and T. D. Abhayapala, "Spatial multizone soundfield reproduction: Theory and design," IEEE Trans. Audio, Speech, Lang. Process., vol. 19, no. 6, pp. 1711-1720, 2011.

[14] T. Betlehem and P. D. Teal, "A constrained optimization approach for multi-zone surround sound," in Proc. IEEE Int. Conf. Acoust., Speech, Signal Process. (ICASSP), Prague, May 2011.

[15] W. Jin and W. B. Kleijn, "Multizone soundfield reproduction in reverberant rooms using compressed sensing techniques," in Proc. IEEE Int. Conf. Acoust., Speech, Signal Process. (ICASSP), Florence, May 2014, pp. $4728-4732$.

[16] W. F. Druyvesteyn and J. Garas, "Personal sound," J. Audio Eng. Soc., vol. 45, no. 9, pp. 685-701, 1997.

[17] T. Betlehem, W. Zhang, M. A. Poletti, and T. D. Abhayapala, "Personal sound zones: Delivering interface-free audio to multiple listeners," IEEE Signal Process. Mag., vol. 32, no. 2, pp. 81-91, 2015.

[18] J.-W. Choi and Y.-H. Kim, "Generation of an acoustically bright zone with an illuminated region using multiple sources," J. Acoust. Soc. Amer., vol. 111, no. 4, pp. 1695-1700, 2002.

[19] Y. Cai, M. Wu, and J. Yang, "Sound reproduction in personal audio systems using the least-squares approach with acoustic contrast control constraint," J. Acoust. Soc. Amer., vol. 135, no. 2, pp. 734-741, 2014.

[20] J. Nocedal and S. J. Wright, Numerical Optimization. New York: Springer, 2006.

[21] S. Koyama, T. Amakasu, N. Ueno, and H. Saruwatari, "Amplitude matching: Majorization-minimization algorithm for sound field control only with amplitude constraint," in Proc. IEEE Int. Conf. Acoust., Speech, Signal Process. (ICASSP), Jun. 2021, pp. 411-415.

[22] D. R. Hunter and K. Lange, "A tutorial on MM algorithms," American Statistician, vol. 58, no. 1, pp. 30-37, 2004.

[23] Y. Sun, P. Babu, and D. P. Palomar, "Majorization-minimization algorithms in signal processing, communications, and machine learning," IEEE Trans. Signal Process., vol. 65, no. 3, pp. 794-816, 2016.

[24] S. Boyd, N. Parikh, E. Chu, B. Peleato, and J. Eckstein, "Distributed optimization and statistical learning via the alternating direction method of multipliers," Found. Trends Mach. Learn., vol. 3, no. 1, pp. 1-122, 2011.

[25] D. Griffin and J. Lim, "Signal estimation from modified short-time fourier transform," IEEE Trans. Acoust., Speech, Signal Process., vol. 32, no. 2, pp. 236-243, 1984.

[26] R. W. Gerchberg and W. O. Saxton, "A practical algorithm for the determination of phase from image and diffraction plane pictures," Optik, vol. 35, pp. 237-246, 1972.

[27] J. Liang, P. Stoica, Y. Jing, and J. Li, "Phase retrieval via the alternating direction method of multipliers," IEEE Signal Process. Lett., vol. 25, no. 1, pp. 5-9, 2018.

[28] Z. Wen, C. Yang, X. Liu, and S. Marchesini, "Alternating direction methods for classical and ptychographic phase retrieval," Inverse Problems, vol. 28, p. 115010, 2012.

[29] T. Narutomi, "Parallel algorithms for large scale computation in science and technology," Ph.D. dissertation, Tohoku University, 1993.

[30] S. Koyama, T. Nishida, K. Kimura, T. Abe, N. Ueno, and J. Brunnström, "MeshRIR: A dataset of room impulse responses on meshed grid points for evaluating sound field analysis and synthesis methods," in Proc. IEEE Int. Workshop Appl. Signal Process. Audio Acoust. (WASPAA), Oct. 2021. 